\title{
YAW DISTURBANCE ATTENUATION BY ROBUST DECOUPLING OF CAR STEERING
}

\author{
J. Ackermann* T. Bünte* \\ * Deutsche Forschungsanstalt \\ für Luft- und Raumfahrt (DLR) Oberpfaffenhofen \\ Institut für Robotik und Systemdynamik \\ 82234 Wessling, Germany \\ e-mail: Juergen.Ackermann@dlr.de
}

\begin{abstract}
Robust decoupling of the lateral and yaw motions of a car has been achieved by feedback of the integrated yaw rate into front wheel steering. In the present paper the yaw disturbance attenuation is analyzed for a generic single-track vehicle model. The frequency limit, up to which yaw disturbances are attenuated, is calculated. For specific vehicle data, it is shown that this control law significantly reduces the influence of yaw disturbances on yaw rate and side-slip angle for low frequencies. This safety advantage is experimentally verified for $\mu$-split braking.
\end{abstract}

Keywords: Automotive control, decoupling problems, robust control, disturbance rejection, sensitivity functions

\section{INTRODUCTION}

Consider the vehicle of Fig. 1.

The input to the system is the front-wheel steering angle $\delta_{f}$, and the output is the yaw rate $r=$ $\dot{\psi}$ measured by a gyro. $\psi$ is the heading angle between an inertial coordinate system $\left(x_{0}, y_{0}\right)$ and the chassis coordinate system $(x, y)$. Uncertain parameters are

(1) the velocity $v=|\vec{v}|>0$,

(2) the cornering stiffness of the tires,

(3) the masses $m_{f}$ and $m_{r}$ at the front and rear axles; it is assumed, however, that the location of the center of gravity $(\mathrm{CG})$ at a distance $\ell_{f}$ from the front axle and $\ell_{r}$ from the rear axle is known, i.e. $m_{f} \ell_{f}=m_{r} \ell_{r}$. The wheelbase is $\ell=\ell_{r}+\ell_{f}$ and the vehicle mass $m=m_{r}+m_{f}$. The assumed ideal mass distribution with $m_{r}=m \ell_{f} / \ell, m_{f}=m \ell_{r} / \ell$ implies the moment of inertia $J_{z}$ w.r.t. a vertical axis through the $\mathrm{CG}$ as

$$
J_{z}=m_{r} \ell_{r}^{2}+m_{f} \ell_{f}^{2}=m \ell_{r} \ell_{f} .
$$

For the case of non ideal mass distribution the reader is referred to (Ackermann, 1994).

For small sideslip angle $\beta$ and small steering angle $\delta_{f}$ the single-track model of car steering is (Mitschke, 1990):

$$
\begin{aligned}
m v(\dot{\beta}+r) & =F_{y}+F_{d} \\
J_{z} \dot{r} & =M_{z}+M_{d} .
\end{aligned}
$$

The lateral force $F_{y}$ and the torque $M_{z}$ around a vertical axis through the $\mathrm{CG}$ are generated by side forces of the tires. Also, a disturbance

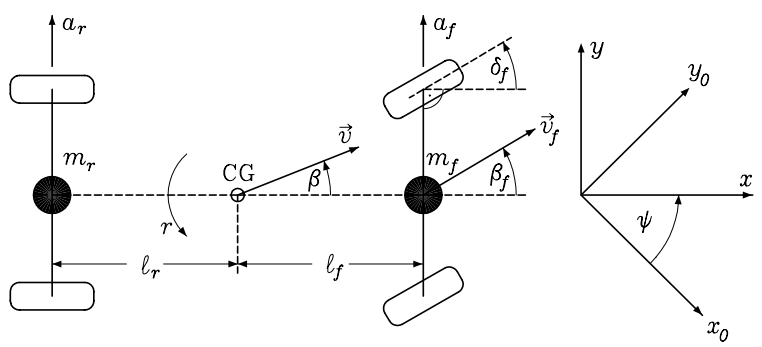

Fig. 1. Vehicle with ideal mass distribution 
force $F_{d}$ and a disturbance torque $M_{d}$ act on the vehicle. Examples are crosswind, lateral road slope, flat tires, and asymmetric braking forces on a slippery road. In particular, an unexpected disturbance torque $M_{d}$ can lead to dangerous driving situations because of the reaction time of the driver, followed by overreactions. Timedelay and high gain may even cause instability of the driver-vehicle feedback system. An automatic control system with feedback of the yaw rate $r$ can react faster and more precisely than a human driver (Ackermann et al., 1993; Ackermann et al., 1996). By integration of $r$ it also provides a relative direction reference with respect to the initial heading of the car before the disturbance. The driver does not have to worry about the automatically controlled yaw motion. He only has to command a lateral acceleration to keep the car, considered as a mass point, on his planned path. He can easily compensate for the lateral disturbance forces $F_{d}$, e.g. from a lateral slope of the road; therefore $F_{d}=0$ was set for simpler notation.

The next sections show the derivation of the disturbance transfer function from $M_{d}$ to the sideslip angle at the front axle and to the yaw rate, first in Section 2 for a conventional car, then in Section 3 for a robustly decoupled car. In Section 4 the steady-state effects and in Section 5 the ratio of the transfer functions (sensitivity function) are analyzed, and the frequency limit of disturbance attenuation is calculated. Section 6 gives an example, and Section 7 shows experimental results.

\section{YAW DISTURBANCE TRANSFER FUNCTION OF THE CONVENTIONAL CAR}

Equations (1) and (2) with $F_{d}=0$ may be written

$$
\left[\begin{array}{c}
m v(\dot{\beta}+r) \\
m \ell_{r} \ell_{f} \dot{r}
\end{array}\right]=\left[\begin{array}{c}
F_{y} \\
M_{z}
\end{array}\right]+\left[\begin{array}{c}
0 \\
M_{d}
\end{array}\right] .
$$

The steering force $F_{y}$ and torque $M_{z}$ are generated by the lateral tire forces $F_{f}\left(\alpha_{f}\right)$ and $F_{r}\left(\alpha_{r}\right)$ via

$$
\left[\begin{array}{c}
F_{y} \\
M_{z}
\end{array}\right]=\left[\begin{array}{cc}
1 & 1 \\
\ell_{f} & -\ell_{r}
\end{array}\right]\left[\begin{array}{c}
F_{f}\left(\alpha_{f}\right) \\
F_{r}\left(\alpha_{r}\right)
\end{array}\right] .
$$

The tire forces depend on the tire sideslip angles $\alpha_{f}$ and $\alpha_{r}$, as illustrated in Fig. 2 for the front wheel. The local velocity vector $\vec{v}_{f}$ forms the (chassis) slip angle $\beta_{f}$ with the car body and the tire slip angle $\alpha_{f}$ with the tire direction; thus

$$
\begin{aligned}
& \alpha_{f}=\delta_{f}-\beta_{f} \\
& \alpha_{r}=\delta_{r}-\beta_{r} .
\end{aligned}
$$

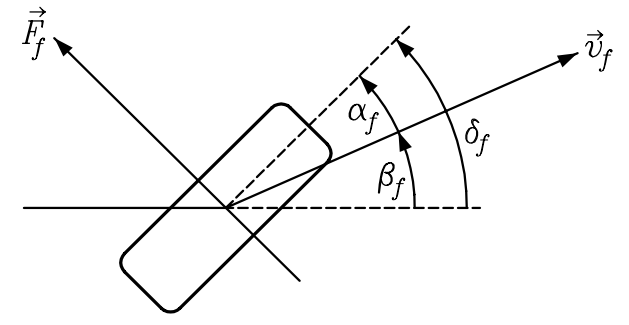

Fig. 2. Variables of the tire model

In this paper rear wheel steering is not used, i.e. $\delta_{r} \equiv 0$. The front sideslip angle $\beta_{f}$ will be introduced as a state variable. The sideslip angles at the $\mathrm{CG}(\beta)$ and at the rear axle $\left(\beta_{r}\right)$ are related to $\beta_{f}$ by the kinematic relations for small angles

$$
\begin{aligned}
\beta & =\beta_{f}-\frac{\ell_{f}}{v} r \\
\beta_{r} & =\beta_{f}-\frac{\ell}{v} r .
\end{aligned}
$$

The tire force characteristics are linearized as

$$
\begin{gathered}
F_{f}\left(\alpha_{f}\right)=c_{f} \alpha_{f} \\
F_{r}\left(\alpha_{r}\right)=c_{r} \alpha_{r}
\end{gathered}
$$

where the cornering stiffnesses $c_{f}$ and $c_{r}$ are uncertain parameters that vary with the road tire contact. It is assumed that $c_{f}=\mu c_{f 0}, c_{v}=\mu c_{r 0}$ where $c_{f 0}$ and $c_{r 0}$ are nominal values for the dry road and $\mu \in\left[\mu^{-} ; 1\right]$ is an uncertain parameter with $\mu^{-}>0$. Further uncertain constant parameters are the vehicle mass $m \in\left[\mathrm{m}^{-} ; \mathrm{m}^{+}\right]$and velocity $v \in\left[v^{-} ; v^{+}\right]$. The model (3), (4) with the above equations substituted becomes

$$
\begin{aligned}
& {\left[\begin{array}{c}
m v\left(\dot{\beta}_{f}-\frac{\ell_{f}}{v} \dot{r}+r\right) \\
m \ell_{r} \ell_{f} \dot{r}
\end{array}\right]=} \\
& \quad=\left[\begin{array}{cc}
1 & 1 \\
\ell_{f} & -\ell_{r}
\end{array}\right]\left[\begin{array}{c}
c_{f}\left(\delta_{f}-\beta_{f}\right) \\
c_{r}\left(-\beta_{f}+\frac{\ell}{v} r\right)
\end{array}\right]+\left[\begin{array}{c}
0 \\
M_{d}
\end{array}\right]
\end{aligned}
$$

and, solving for $\dot{\beta}_{f}$ and $\dot{r}$,

$$
\begin{aligned}
{\left[\begin{array}{c}
\dot{\beta}_{f} \\
\dot{r}
\end{array}\right]=} & {\left[\begin{array}{cc}
\frac{1}{m_{f} v} & 0 \\
\frac{1}{m_{f} \ell} & \frac{-1}{m_{r} \ell}
\end{array}\right]\left[\begin{array}{c}
c_{f}\left(\delta_{f}-\beta_{f}\right) \\
c_{r}\left(-\beta_{f}+\frac{\ell}{v} r\right)
\end{array}\right]-} \\
& -\left[\begin{array}{l}
1 \\
0
\end{array}\right] r+\left[\begin{array}{c}
\frac{1}{m_{f} v \ell} \\
\frac{m_{r}+m_{f}}{m_{r} m_{f} \ell^{2}}
\end{array}\right] M_{d}
\end{aligned}
$$

where the front and rear masses $m_{f}=m \ell_{r} / \ell$ and $m_{r}=m \ell_{f} / \ell$ have been substituted to replace $m, \ell_{r}$ and $\ell_{f}$. The state equations of the system are then 


$$
\begin{array}{r}
{\left[\begin{array}{c}
\dot{\beta}_{f} \\
\dot{r}
\end{array}\right]=\left[\begin{array}{cc}
\frac{-c_{f}}{m_{f} v} & -1 \\
\frac{1}{\ell}\left(\frac{c_{r}}{m_{r}}-\frac{c_{f}}{m_{f}}\right) & \frac{-c_{r}}{m_{r} v}
\end{array}\right]\left[\begin{array}{c}
\beta_{f} \\
r
\end{array}\right]+} \\
+\frac{c_{f}}{m_{f}}\left[\begin{array}{c}
\frac{1}{v} \\
\frac{1}{\ell}
\end{array}\right] \delta_{f}+\frac{1}{m_{f} \ell}\left[\begin{array}{c}
\frac{1}{v} \\
\frac{m_{r}+m_{f}}{m_{r} \ell}
\end{array}\right] M_{d}
\end{array}
$$

Laplace-transformation of (10) yields

$$
\begin{aligned}
& {\left[\begin{array}{cc}
s+\frac{c_{f}}{m_{f} v} & 1 \\
\frac{1}{\ell}\left(\frac{c_{f}}{m_{f}}-\frac{c_{r}}{m_{r}}\right) & s+\frac{c_{r}}{m_{r} v}
\end{array}\right]\left[\begin{array}{c}
\beta_{f}(s) \\
r(s)
\end{array}\right]=} \\
& =\frac{c_{f}}{m_{f}}\left[\begin{array}{l}
\frac{1}{v} \\
\frac{1}{\ell}
\end{array}\right] \delta_{f}(s)+\left[\begin{array}{c}
\frac{1}{v} \\
\frac{m_{r}+m_{f}}{m_{r} \ell}
\end{array}\right] \frac{M_{d}(s)}{m_{f} \ell} .
\end{aligned}
$$

Solve (11) for $\beta_{f}(s)$ and $r(s)$ and let $\delta_{f}(s) \equiv 0$.

$$
\begin{gathered}
{\left[\begin{array}{c}
\beta_{f}(s) \\
r(s)
\end{array}\right]=\frac{1}{D(s) m_{f} \ell}\left[\begin{array}{c}
B(s) \\
R(s)
\end{array}\right] M_{d}(s)} \\
B(s)=\frac{1}{v}\left(s+\frac{c_{r}}{m_{r} v}-\frac{\left(m_{r}+m_{f}\right) v}{m_{r} \ell}\right) \\
R(s)=\frac{m_{r}+m_{f}}{m_{r} \ell}\left(s+\frac{c_{r}+c_{f}}{v\left(m_{r}+m_{f}\right)}\right) \\
D(s)=\left(s+\frac{c_{f}}{m_{f} v}\right)\left(s+\frac{c_{r}}{m_{r} v}\right)- \\
\quad-\frac{1}{\ell}\left(\frac{c_{f}}{m_{f}}-\frac{c_{r}}{m_{r}}\right)
\end{gathered}
$$

\section{YAW DISTURBANCE TRANSFER FUNCTION OF THE ROBUSTLY DECOUPLED CAR}

Robust decoupling (Ackermann et al., 1993) is based on the requirement that the yaw rate $r$ is made unobservable from the lateral acceleration $a_{f}$ at the front axle. This goal is achieved by the feedback control law

$$
\dot{\delta}_{f}=-r+F \delta_{L} .
$$

It is assumed here that the steering wheel input $\delta_{L}$ of the driver is zero.
Substitute $\delta_{f}(s)=-r(s) / s$ into (11):

$$
\begin{gathered}
{\left[\begin{array}{cc}
s+\frac{c_{f}}{m_{f} v} & 1+\frac{c_{f}}{m_{f} v s} \\
\frac{1}{\ell}\left(\frac{c_{f}}{m_{f}}-\frac{c_{r}}{m_{r}}\right) & s+\frac{c_{r}}{m_{r} v}+\frac{c_{f}}{m_{f} \ell s}
\end{array}\right] .} \\
\cdot\left[\begin{array}{c}
\beta_{f}(s) \\
r(s)
\end{array}\right]_{d e c}=\left[\begin{array}{c}
\frac{1}{v} \\
\frac{m_{r}+m_{f}}{m_{r} \ell}
\end{array}\right] \frac{M_{d}(s)}{m_{f} \ell} .
\end{gathered}
$$

Solve (14) for $\beta_{f d e c}(s)$ and $r_{d e c}(s)$.

$$
\begin{gathered}
{\left[\begin{array}{c}
\beta_{f d e c}(s) \\
r_{d e c}(s)
\end{array}\right]=\frac{1}{D_{d e c}(s) m_{f} \ell}\left[\begin{array}{l}
B_{d e c}(s) \\
R_{d e c}(s)
\end{array}\right]} \\
B_{d e c}(s)=s B(s)-\frac{c_{f}}{m_{r} v \ell} \\
R_{d e c}(s)=s R(s) \\
D_{d e c}(s)=s D(s)+\frac{c_{f}}{m_{f} \ell}\left(s+\frac{c_{r}}{m_{r} v}\right) \\
\quad=\left(s+\frac{c_{f}}{m_{f} v}\right)\left[s^{2}+\frac{c_{r}}{m_{r} v}\left(s+\frac{v}{\ell}\right)\right] .
\end{gathered}
$$

The control law (13) provides a reference for the inertial front wheel orientation $\psi+\delta_{f}$, where $r=\dot{\psi}$. Then

$$
\begin{aligned}
\psi(t) & =\psi\left(t_{0}\right)+\int_{t_{0}}^{t} r(\tau) d \tau \\
\psi(t) & =\psi\left(t_{0}\right)+\delta_{f}\left(t_{0}\right)-\delta_{f}(t) \\
\psi(t)+\delta_{f}(t) & =\psi\left(t_{0}\right)+\delta_{f}\left(t_{0}\right) .
\end{aligned}
$$

In the decoupled car the inertial orientation of the front wheels is not influenced by a disturbance torque $M_{d}$.

\section{STEADY-STATE EFFECT OF YAW DISTURBANCES}

For a step disturbance input, $M_{d}(s)=1 / s$, the conventional car has the steady-state response

$$
\begin{aligned}
\beta_{f s t} & =\frac{B(0)}{D(0) m_{f} \ell}=\frac{c_{r} \ell-\left(m_{r}+m_{f}\right) v^{2}}{c_{f} c_{r} \ell^{2}-v^{2} \ell\left(c_{f} m_{r}-c_{r} m_{f}\right)} \\
r_{s t} & =\frac{R(0)}{D(0) m_{f} \ell}=\frac{\left(c_{r}+c_{f}\right) v}{c_{f} c_{r} \ell^{2}-v^{2} \ell\left(c_{f} m_{r}-c_{r} m_{f}\right)} .
\end{aligned}
$$

The robustly decoupled car has the steady-state response 


$$
\begin{gathered}
\beta_{f s t, d e c}=\frac{B_{d e c}(0)}{D_{d e c}(0) m_{f} \ell}=\frac{-1}{c_{r} \ell} \\
r_{s t, d e c}=\frac{R_{d e c}(0)}{D_{d e c}(0) m_{f} \ell}=0 .
\end{gathered}
$$

The yaw rate goes to zero, i.e. the car has a constant heading angle as a steady-state response to a step yaw disturbance.

\section{DISTURBANCE ATTENUATION RATIOS; FREQUENCY LIMIT}

Define

$$
\begin{aligned}
& \rho_{\beta}(s)=\frac{B_{d e c}(s) D(s)}{D_{d e c}(s) B(s)} \\
& \rho_{r}(s)=\frac{R_{d e c}(s) D(s)}{D_{d e c}(s) R(s)}=\frac{s D(s)}{D_{d e c}(s)} \\
& \rho_{\beta}(s)=\left(1-\frac{c_{f}}{m_{r} v \ell s B(s)}\right) \rho_{r}(s)
\end{aligned}
$$

as disturbance attenuation ratios (sensitivity functions). The influence of the yaw disturbances on $\beta_{f}$ or $r$ is reduced for all frequencies for which $\left|\rho_{\beta}(j \omega)\right|<1$ or $\left|\rho_{r}(j \omega)\right|<1$, respectively.

Let $a=c_{f} / m_{f} \quad b=c_{r} / m_{r}$, then

$$
\begin{gathered}
\rho_{r}(s)=\frac{[(s+a / v)(s+b / v)-(a-b) / \ell] s}{(s+a / v)\left[s^{2}+(s+v / \ell) b / v\right]} \\
=\frac{\left[v^{2} \ell s^{2}+v \ell(a+b) s+a b \ell-(a-b) v^{2}\right] s}{(v s+a)\left[v \ell s^{2}+(\ell s+v) b\right]} \\
\left|\rho_{r}(j \omega)\right|^{2}=\rho_{r}(j \omega) \rho_{r}(-j \omega)= \\
=\frac{\left[a b \ell-v^{2} \ell \omega^{2}-(a-b) v^{2}\right]^{2}+v^{2} \ell^{2}(a+b)^{2} \omega^{2}}{\left(v^{2}+(a / \omega)^{2}\right)\left[\left(-v \ell \omega^{2}+v b\right)^{2}+\ell^{2} b^{2} \omega^{2}\right]} \\
\rho_{r}(0)=0
\end{gathered}
$$

$\left|\rho_{r}(j \omega)\right|^{2}<1$ if and only if

$$
\begin{aligned}
& \left(v^{2} \omega^{2}+a^{2}\right)\left[v^{2}\left(-\ell \omega^{2}+b\right)^{2}+\ell^{2} b^{2} \omega^{2}\right]- \\
& -\left[a b \ell-v^{2} \ell \omega^{2}-(a-b) v^{2}\right]^{2} \omega^{2}- \\
& -v^{2} \ell^{2}(a+b)^{2} \omega^{4}>0 .
\end{aligned}
$$

which may be simplified to

$$
\omega^{4}-\left(\frac{2 b-a}{2 \ell}-\frac{b^{2}}{v^{2}}\right) \omega^{2}-\frac{a b^{2}}{2 \ell v^{2}}>0 .
$$

The frequency limit is
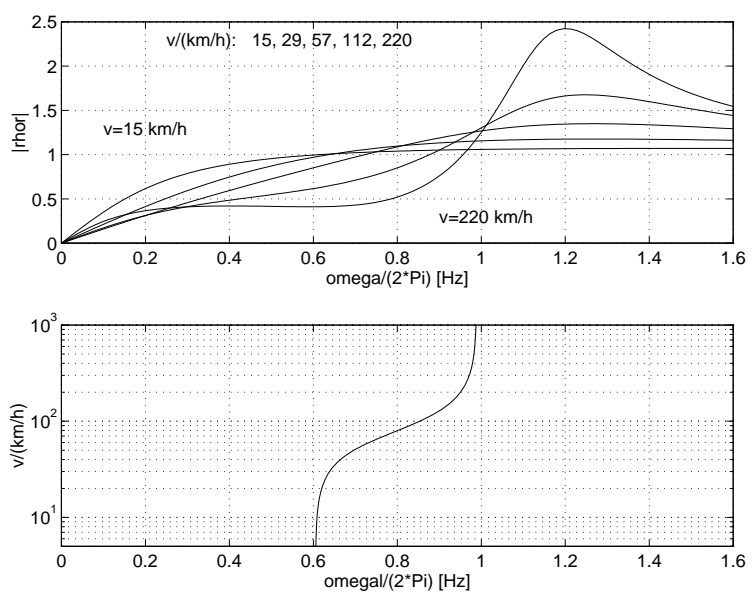

Fig. 3. Disturbance attenuation (sensitivity function) and frequency limit for varying velocity

$$
\begin{aligned}
\omega_{\ell}^{2} & =c+\sqrt{c^{2}+a b^{2} / 2 \ell v^{2}} \\
c & =\frac{2 b-a}{4 \ell}-\frac{b^{2}}{2 v^{2}} .
\end{aligned}
$$

The yaw disturbances are attenuated for frequencies

$\omega<\omega_{\ell}$, and amplified for $\omega>\omega_{\ell}$.

\section{EXAMPLE}

Consider a car with the following parameters

$\ell_{f}=1.514 \mathrm{~m}, \quad \ell_{r}=1.323 \mathrm{~m}, m=1916 \mathrm{~kg}$,

$c_{f}=49400 \mathrm{~N} / \mathrm{rad}, \quad c_{r}=103800 \mathrm{~N} / \mathrm{rad}$

and uncertain velocity $v \in[15 ; 220] \mathrm{km} / \mathrm{h}$.

Fig. 3 shows the disturbance attenuation at five velocity values. The frequency limit in the lower figure is between 0.6 and 1 Hertz.

\section{EXPERIMENTAL RESULTS}

A periodic disturbance torque $M_{d}$ is not typical for car driving. A much harder test is a step disturbance $M_{d}(s)=M / s$. This is generated experimentally in the $\mu$ split test shown in Fig. 4 . The left-hand column of pictures, taken at 1second intervals, shows the experiment with a conventional car. The car drives with its right-hand wheels (left-hand side of the figure) on waterflooded tiles with a friction coefficient $\mu \approx 0.1$, and with the left-hand wheels on wet asphalt with a friction coefficient $\mu \approx 0.9$. At an initial velocity of $80 \mathrm{~km} / \mathrm{h}$ the driver brakes and keeps his steering wheel straight. In the second picture the beginning of skidding is recognizable; only one second later the car has all four tires on asphalt in a safe situation. In normal driving on an icy road it takes the driver just this second to react, and that may be too late. The right-hand column shows the 

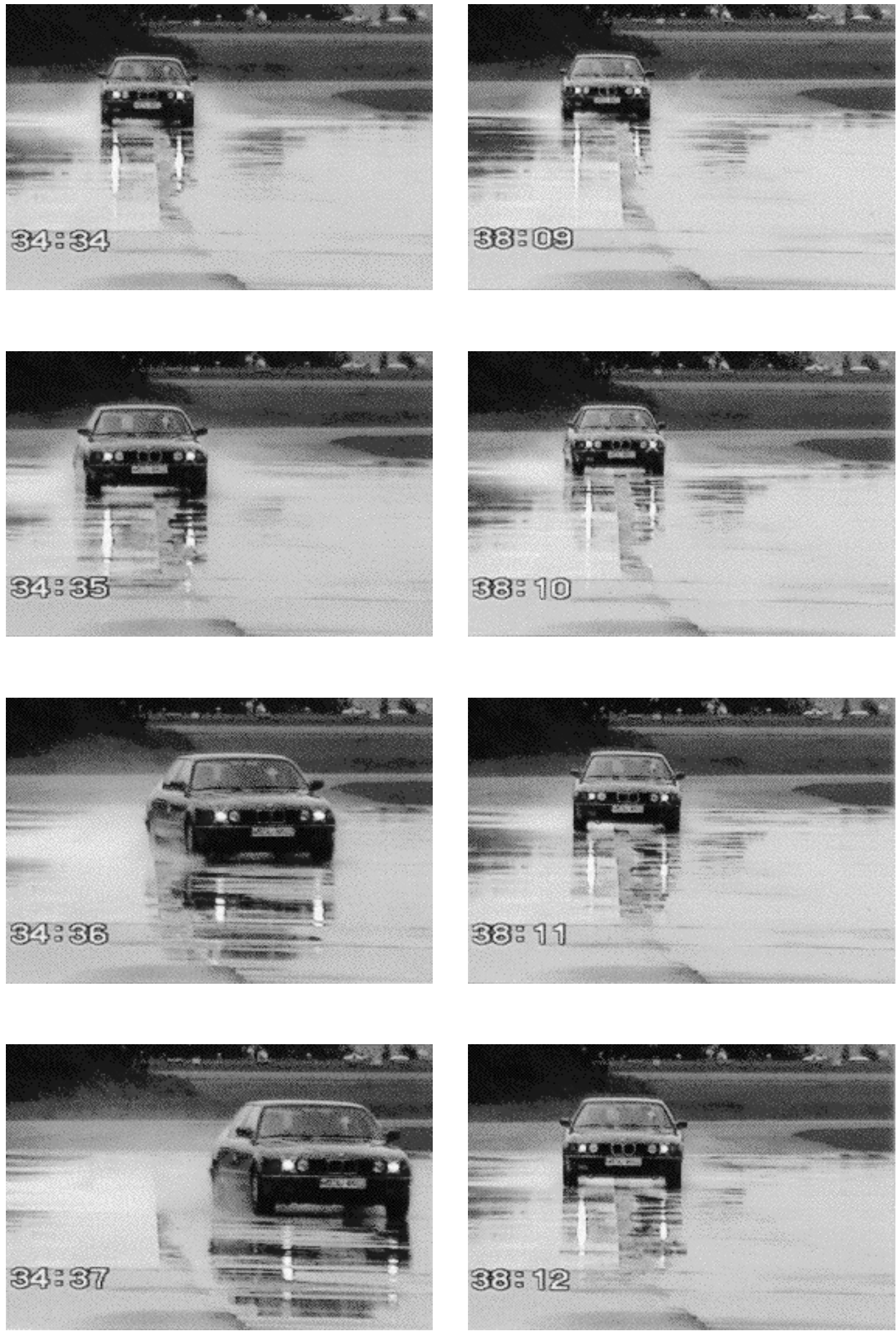

Fig. 4. $\mu$-split-braking at $80 \mathrm{~km} / \mathrm{h}$, left: conventional vehicle, right: robustly decoupled vehicle 

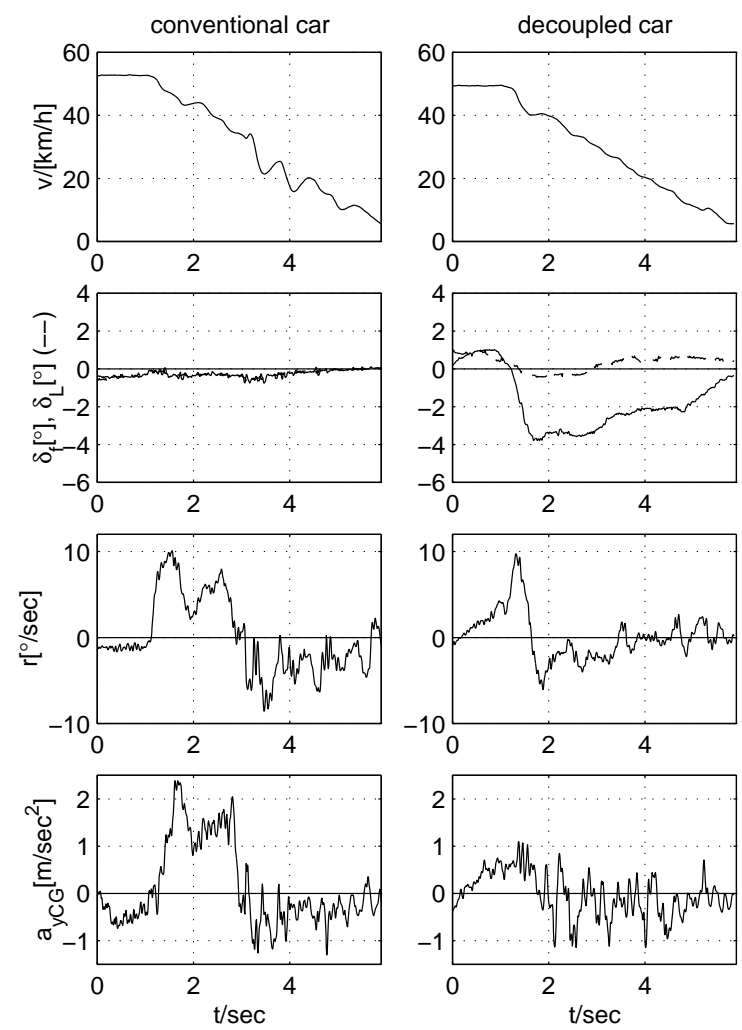

Fig. 5. Experimental data for $\mu$-split braking with inital velocity $v \approx 50 \mathrm{~km} / \mathrm{h}$

same experiment with the robustly decoupled vehicle. In the second picture the situation is similar to that of the conventional car. But now the yaw rate is measured and the controller steers the car quickly back to the original heading angle. The car remains straight with yaw rate back to zero, see (18), until the complete stop. This spectacular safety improvement is not only obtained at nominal speed, load and friction coefficients but with perfect robustness against changes in these parameters.

Fig. 5 shows a comparison of experimental data for $\mu$-split braking for the conventional car (left) and for the decoupled car (right). The velocity $v$ shows the occurrence of the $M_{d}$-step by braking and the speed reduction. The angle $\delta_{L}$ (handwheel angle $\times$ steering gear ratio) is kept close to zero in both experiments, in the decoupled car a front wheel steering angle $\delta_{f}$ from the controller (13) occurs in response to the initial peak in the yaw rate $r$. In contrast to the conventional vehicle, feedback quickly reduces $r$ and changes its sign such that $\psi(t)=\psi\left(t_{0}\right)$ according to (17). The lateral acceleration $a_{y C G}=v(\dot{\beta}+r)$ at the center of gravity shows a significant reduction by the decoupling controller.

\section{CONCLUSIONS}

The robustly decoupling control law for cars attenuates yaw disturbances, e.g. from crosswinds, flat tires, or asymmetric braking forces for low frequencies. The frequency limit is calculated for generic vehicle parameters. The effect for a specific car is shown by the calculated disturbance attenuation ratio and by $\mu$-split braking tests.

\section{REFERENCES}

Ackermann, J. (1994). Robust decoupling of car steering dynamics with arbitrary mass distribution. In: Proc. American Control Conference. Vol. 2. Baltimore, USA. pp. 1964-1968.

Ackermann, J., A. Bartlett, D. Kaesbauer, W. Sienel and R. Steinhauser (1993). Robust control: Systems with uncertain physical parameters. Springer. London.

Ackermann, J., T. Bünte, W. Sienel, H. Jeebe and K. Naab (1996). Driving safety by robust steering control. In: Proc. Int. Symposium on Advanced Vehicle Control. Aachen, Germany. Mitschke, M. (1990). Dynamik der Kraftfahrzeuge. Vol. C. Springer. Berlin. 